\title{
An Improved LQI- based Link Quality Estimation Mechanism for Wireless Sensor Networks
}

\author{
Wei Chen, Jian Luo \\ School of Information Science and Engineering \\ Hunan University \\ Changsha, China \\ cecilia327@hnu.edu.cn
}

\begin{abstract}
An accurate and efficient link quality estimation method is crucial for the performance of the upper layer routing protocol, flow distribution and topology control algorithms in WSNs (Wireless Sensor Networks). Based on the analysis of the cause for packet loss and bit error, an improved LQI (Link Quality Indicator)-based link quality estimation mechanism called imLQI is introduced, which takes into account the LQI values of the unsuccessfully received packets when calculates the average LQI values. The accuracy and efficiency of imLQI has been proved by diverse experiments running on real testbed. On the basic of the mechanism, this paper also develops an LQI-based link quality estimation model, and multiple measurements have been executed to validate the correctness of this model.
\end{abstract}

Keywords-wireless sensor networks; link quality estimation; link quality indicator; link quality measurements

\section{INTRODUCTION}

Wireless Sensor Networks are formed by energy-limited, memory-limited and low power sensor nodes in a selforganizing pattern. Being influenced by path loss, fading, noise, multipath effect, external interference and etc., the transmission quality between two nodes in WSN is unstable, and the wireless links perform unstably with packet error/loss and vulnerability to the environment. Hence, reliable, real-time and stable link quality mechanisms are crucial to WSN: poor LQEs (Link Quality Estimators) may cause a $200 \%$ or more drop in network throughput [1]. Meantime, upper layer protocols require LQE to maintain correct transitions and overcome unreliable links [2], and the topology control algorithms choose good links via link quality estimation mechanisms to sustain stable topology [3]. In addition, high-quality links reduce energy consumption by decreasing retransmission attempts, and therefore prolong the network lifetime.

The existing link quality estimation methods can be divided into two categories: software-based and hardwarebased [4].

The software-based methods use PRR (Packet Receive Ratio) within a time window to estimate link quality. In general, it requires nodes to broadcast plenty of probes in a large time window to estimate link quality directly and exclusively by calculating PRR, so it may not respond swiftly to link status changes. Meanwhile, the redundant probes may increases the burden of nodes or even cause congestion. Some studies use Kalman filter to reduce the window size to calculate PRR [5], but those algorithms are too complex to be adapted in WSN.

The hardware-based methods estimate link quality on the basic of 3 metrics which can be provided by communication chips: RSSI (Received Signal Strength Indicator), LQI (Link Quality Indicator) and SNR (Signal-Noise Ratio). Compared with software-based methods, hardware-based methods require less overhead; hence better sensitivity to link quality changes. Therefore, hardware-based LQEs are widely used in WSN. Srinivasan et al. [6] suggested that the mean value of LQI (hereinafter referred to as mean LQI) computed over about 120 packets is correlated to PRR. Zhu et al.'s study [7] showed that compared to RSSI and SNR, LQI reflects link quality more accurately. Nowadays, many researches concerning LQEs based on LQI have been done. Zhu et al. [8] proposed a link quality estimation model based on LQI, and they pointed out that the relationship between the change of mean value of LQI and PRR is Gaussian, so link quality can be inferred by LQI through this model. Boano et al. [9] designed a fast and reliable LQE, called Triangle Metric, which geometrically combines the information of PRR, LQI and SNR, and they managed to estimate link quality accurately within 10 packets. Yuan et al. [10] improved the performance of ETX (Expected Number of Transmission) by adding LQI into the algorithm computing ETX, and then presented a new cumulative link quality routing algorithm I WSN.

However, traditional LQEs based on LQI only take into account the LQI values of successfully received packets when computing mean LQI, and it may lead to serious bias. For instance, assuming link A only received one packet whose LQI value is 90 and lost every other packets, while link B only lost one packets and received every other packets whose mean LQI is 80 , the system will select link A as a better link according to the traditional algorithm, yet actually link A outperforms link B. Therefore, to compensate the drawback of traditional method, this paper proposes an improved LQI -based link quality estimation mechanism called imLQI (improved LQI).

\section{Motivation}

In this section, we will introduce traditional method calculating mean LQI and different situations of packet loss. 


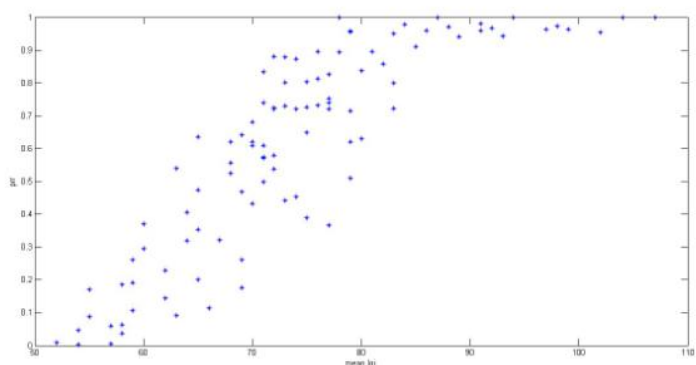

Figure 1. Relationship between mean LQI and PRR computing by traditional method

\section{A. Traditional Mean LQI Calculation}

In WSN, data are delivered hop by hop from source nodes to sink node. Assuming node A and node B are neighbors and the time window during the transmission between $\mathrm{A}$ and $\mathrm{B}$ is $\left[\mathrm{t}_{0}, \mathrm{t}_{1}\right]$, then the equation to compute mean LQI in $\left[\mathrm{t}_{0}, \mathrm{t}_{1}\right]$ is as follows:

$$
\overline{L Q I}=\frac{\sum_{g=1}^{Z n} l q i_{g}}{m}
$$

$\overline{L Q I}$ means the mean LQI in $\left[\mathrm{t}_{0}, \mathrm{t}_{1}\right]$, and $m$ means the count of successfully received packets in $\left[\mathrm{t}_{0}, \mathrm{t}_{1}\right]$.

In practical measurement, we set the packet length to $36 \mathrm{~KB}$, data transmission rate to $250 \mathrm{kbps}$, IPI ( Inter-Packet Interval) to 30PPS (Packet per Second) and transmission power to $-10 \mathrm{dBm}$, and we show the relationship between $\overline{L Q I}$ and PRR in Fig. 1.

According to Fig. 1, when mean LQI is larger than 83, PRR is larger than 0.9, which means link is in good quality; when mean LQI belongs to [56, 83], mean LQI is poorly correlated to PRR, and a single reading of mean LQI corresponds to several PRR readings. Hence, the estimation result conducted by the traditional method can't distinguish respectively good links from bad ones, so there is room for improvement for the traditional method.

\section{B. Different Situations of Packet Loss}

Some study shows that, when taking no account of nodes' death, power-off, sleep or malfunction, there are mainly 3 reasons for packet loss [13]: 1 . The received signal strength is lower than the sensitivity threshold at the receiver, so no data will be received; 2 . The sync bits in packets are interfered during transmission, so the receiver can't be synchronized with the incoming packets; 3 . Bit errors occur in packets due to interference, so the packets can't pass the CRC after being decoded.

After many measurements and data statistics taken, we found that there are mainly 2 types of packet loss phenomena during transmission, as shown in Fig. 2.

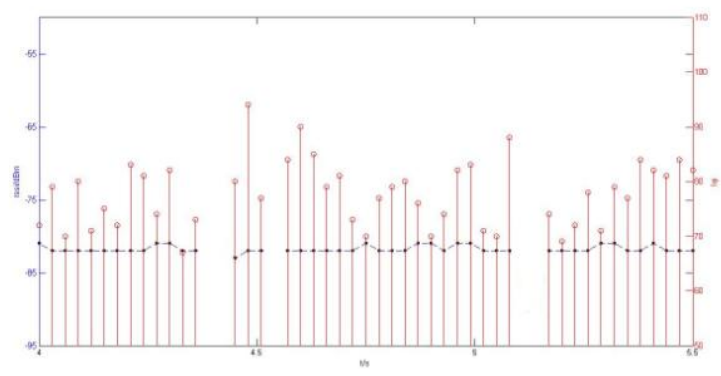

(a)

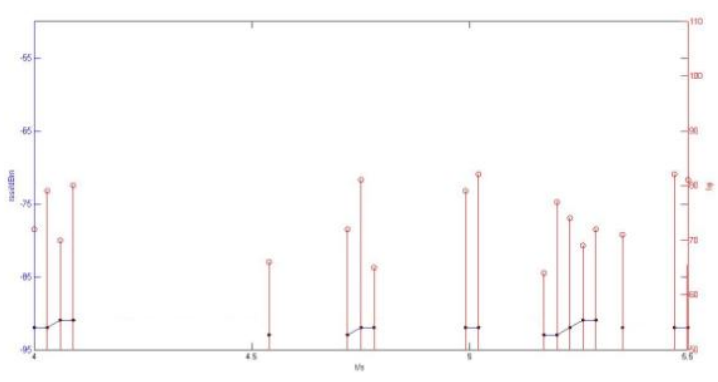

(b)

Figure 2. Relationship between RSSI and LQI within a time window

In Fig. 2(a), packet loss only occurs a few times, inconsecutively, and RSSI values change around $-82 \mathrm{dBm}$ with a range less than $\pm 2 \mathrm{dBm}$; LQI fluctuates severely in $[65,94]$ with the majority are in $[70,88]$. In Fig. 2(b), packet loss occurs consecutively, and the RSSI values in the successfully received packets change around $-92 \mathrm{~dB}$ with a range less than $\pm 2 \mathrm{dBm}$; LQI fluctuates severely in $[57,96]$ with the majority are in $[62,75]$. After comparing the 2 figures above, we can infer that the link quality shown in Fig. 2(a) is better than that in Fig. 2(b).

To further analyze, we found that the RSSI values of packets received before/after the lost packets in Fig. 2(a) remain stable and higher than the sensitivity threshold, which is $-95 \mathrm{dBm}$ under our experiment settings [15]. Therefore, there may be 2 causes for the packet loss in Fig. 2(a): the sync bites in packets are interfered during transmission, or bit errors occur in packets due to interference. As for Fig. 2(b), the packet loss may be caused by the received signal attenuating below the sensitivity threshold.

\section{DESIGNING IMLQI}

In this section, we will discuss the specifics in imLQI design on the basis of the 3 packet loss situations described in section II. 


\section{A. Dealing with Packets fail CRC}

During the transmission process, some packets may be interfered by occasional factors, leading to bit errors, so they will fail passing CRC at the receiver. In fact, the LQI values in those packets reveal link quality changes more explicitly than the successfully received packets. Hence, the LQI values of the packets which fail CRC should particularly be taken into the compute of mean LQI.

To attain the LQI values of the packets which can't pass CRC, we switch off the CRC mechanism by reprogramming the receiver, so that packets with bit errors can be decoded by the receiver.

\section{B. Dealing with Packets with Error Sync Bits}

The general phenomenon of packet loss caused by error sync bits is: the RSSI values of packets successfully received in time windows before and after the packet loss are more than $3 \mathrm{dBm}$ higher than the sensitivity threshold. For this kind of lost packets, we calculate their LQI values according to 3 specific situations:

- If $n 1$ packets are lost during [0,tn1] (0 is the time when the transmission starts) and packets from No.n1+1 to No.m1 are successfully received afterwards, then the mean LQI, $\overline{l q i_{w 1}}$, during the time window $\left[t_{n l+1}, t_{w l}\right](w 1 \leq m 1)$ is

$$
\overline{l q i_{W 1}}=\frac{\sum_{i=n 1+1} l q i_{i}^{i}}{w 1-n 1}
$$

The mean LQI is a statistic-based value whose error is inversely proportional to the size of time window. In order to balance sensitivity and accuracy of link quality estimation, the size of $w 1$ in our work is an empirical value based on multiple experiments:

$$
\left\{\begin{array}{lr}
w 1=m 1, & n 1+1 \leq m 1<n 1+21 ; \\
w 1=20, & m 1 \geq n 1+21 .
\end{array}\right.
$$

Because that the packet lost in this situation is caused by error sync bits, so we can safely infer that the link quality in $\left[0, t_{n l}\right]$ is approximately the same as that in $\left[t_{n l+1}, t_{w l}\right]$. Assuming the mean LQI in $[0$, $\left.t_{n 1}\right]$ is $\overline{l q i_{1}}$, then there is an equation: $\overline{l q i_{1}}=\overline{l q i_{111}}$.

- If $\mathrm{n} 3-\mathrm{n} 2+1$ packets are lost during $\left[t_{n 2}, t_{n 3}\right]$ in the middle of transmission and packets from No.m2 to No.(n2-1) before that are received, then the mean LQI, $\overline{l q i_{w 2}}$, during $\left[t_{w 2}, t_{n 2-1}\right]\left(w^{2} \leq n 2-1\right)$ is

$$
\overline{l q i_{w 2}}=\frac{\sum_{i=122}^{m} l q i_{i}^{i}}{n 2-w 2}
$$

where

$$
\left\{\begin{array}{lr}
w 2=m 2, & n 2-1 \leq m 2<n 2-21 ; \\
w 2=20, & m 2 \geq n 2-21 .
\end{array}\right.
$$

After the packet loss, packets from No.(n3+1) to No.m3 are delivered, then the mean LQI, $\overline{l q i_{w a}}$, during $\left[t_{n 3+1}, t_{w 3}\right]\left(w^{3} \leq m 3\right)$ is

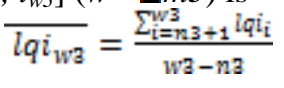

where

$$
\left\{\begin{array}{lr}
w 3=m 3, & n 3+1 \leq m 1<n 3+21 ; \\
w 3=20, & m 3 \geq n 3+21 .
\end{array}\right.
$$

Assuming the mean LQI during $\left[t_{n 2}, t_{n 3}\right]$ is $\overline{\operatorname{lq} i_{2}}$, then

$$
\overline{\operatorname{lq} i_{2}}=\frac{\overline{\operatorname{lq} i_{W 2}}+\overline{l q i_{w 3}}}{2}
$$

- If packet loss occurs in $\left[t_{n 4}, t_{N}\right]\left(t_{N}\right.$ is the time when transmission stops) and $N-n 4+1$ packets are lost, then the mean LQI, $\overline{l q i_{w 4}}$, during $\left[t_{w 4}, t_{n 4-1}\right]$ $(w 4 \leq n 4-1)$ is

$$
\begin{aligned}
& \qquad \overline{l q i_{a}}=\overline{l q i_{w 4}}=\frac{\sum_{i=14}^{n 4-1} \mathbb{I q i _ { i }}}{m 4-144} \\
& \text { where } \\
& \left\{\begin{array}{lr}
w 4=m 4, & n 4-1 \leq m 4<n 4-21 ; \\
w 4=20, & m 4 \geq n 4-21 .
\end{array}\right.
\end{aligned}
$$

If the first/last transmitted packet is lost, we assume its LQI value is the same as that of the packet transmitted after/before it, and if a single packet is lost during transmission, we compute its LQI value by taking the average of the LQI values in the packets sent before and after the lost one.

\section{Dealing with Packets with Low Signal}

If the signal in a packet is lower than the sensitivity threshold (sensitivity threshold varies along with different environment, and the sensitivity threshold in our experiments is $-95 \mathrm{dBm}$ according to the datasheet of CC1100), the receiver can't detect the packet. The general phenomenon of this type of packet loss is: the RSSI values of packets successfully received in time windows before and after the packet loss are less than $3 \mathrm{dBm}$ higher than the sensitivity threshold.

RSSI is a fairly stable value whose deviation is usually small [6], so this type of packet loss indicates that the signals of packets on the link are low and near the sensitivity threshold. Under this condition, the link quality is usually poor with PRR approximately equal to 0 . There are studies show that when PRR on a link is near to 0 , the mean LQI is near to 50 [7]. Therefore, we estimate the mean LQI of packets whose signals are lower than the sensitivity threshold to be 50 .

\section{Performance Evaluation}

In this section, we will specifically discuss the experiments and empirical data. We run multiple sets of experiments on testbed to examine the performance of imLQI, and built a link quality estimation model based on imLQI according to the measured data. By comparing the theoretical result and actual measured result, we testified the correctness and effectiveness of the estimation model we built.

\section{A. Experiment Setup}

The testbed for WSN measurement was composed by Atmega 128A and CC1100, which were manufactured by Texas Instruments, and embedded with TinyOS 2.1 operation system designed by University of California, Berkeley. The data collection and analysis system was Cbased. All the experiments were executed in an empty hallway to avoid being influenced by occasional obstacles, electromagnetic interference or other adverse factors. The setting of experiment is shown in Fig. 3. 


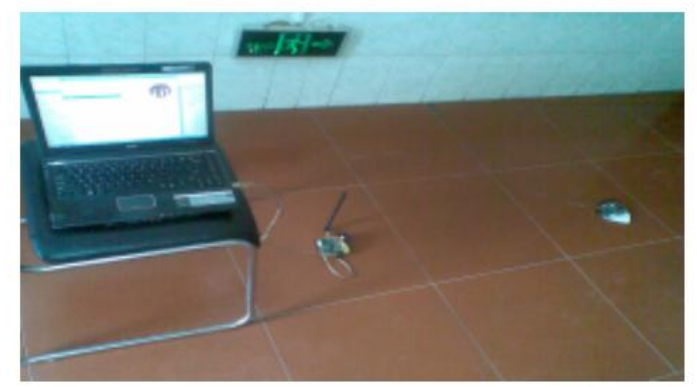

Figure 3. Experimental environment

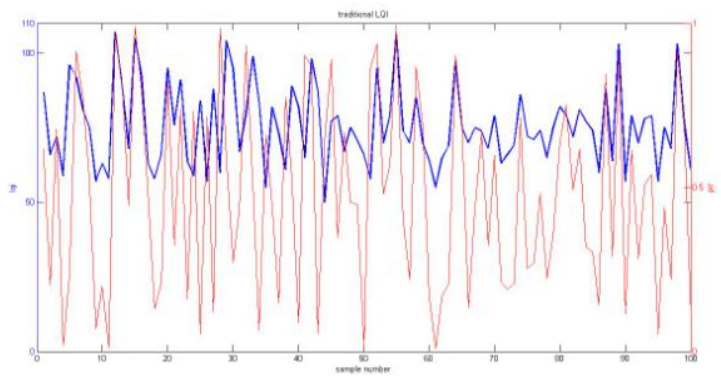

(a)

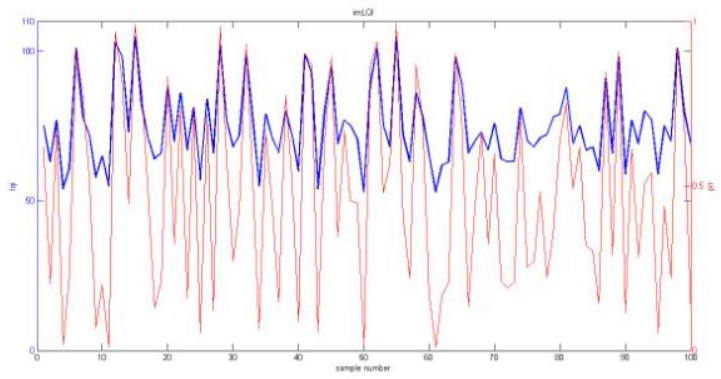

(b)

Figure 4. Relationships of mean LQI and PRR over time

The traffic pattern was generated as follows. The receiver periodically (every 90s) received a burst of 500 consecutive packets from the transmitter, which transmitted one by one. Packets were transmitted at $433 \mathrm{MHZ}$ with a payload length of 36 bytes. For each of the received packets, the receiver stores the LQI values. By using the corresponding and sequence number, the PRR over subsequent burst was computed.

\section{B. Experiment Results}

To examine the link quality estimation performance of imLQI, we study the relationships between PRR and LQI derived from traditional LQI and imLQI over 150min, as

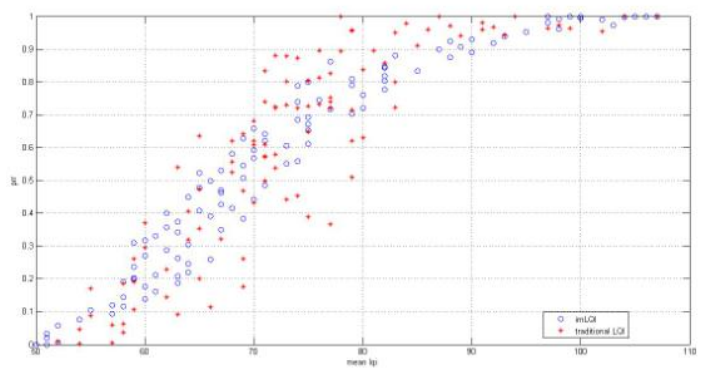

Figure 5. Scatter diagrams comparison of mean LQI and PRR

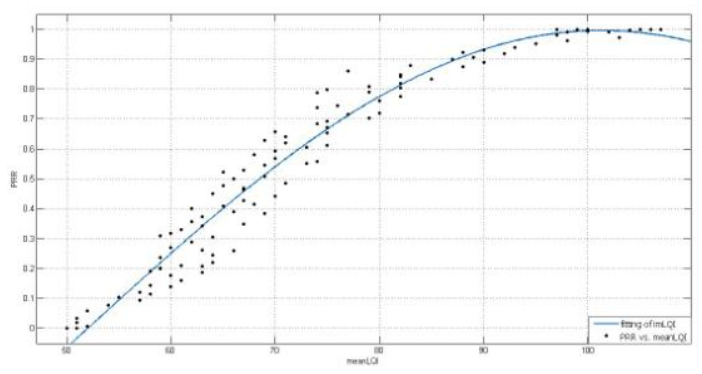

Figure 6. Fitting of mean LQI and PRR of imLQI

shown in Fig.4. In Fig 4(a), which describes the relationship between PRR and mean LQI derived from traditional LQI, the trend of mean LQI severely mismatches that of PRR. On the other hand, the plot of mean LQI basically changes along with the plot of PRR in Fig. 4(b), which describes the relationship between PRR and mean LQI derived from imLQI. To further testify the superiority of imLQI compared with traditional method, we plot the scatter diagram of the relationship between PRR and mean LQI, as shown in Fig. 5. In Fig. 5, mean LQI computed by imLQI is more correlated to mean LQI computed by traditional method when PRR is in [0.1, 0.9], which is known as the "transitional region" [4]. When PRR is in [0, 0.1], the "disconnect region" [4], and [0.9, 1], the "connected region" [4], the correlation of mean LQI and PRR of the 2 methods are nearly the same. We use Pearson Correlation to quantize the correlation of mean LQI and PRR computed by imLQI:

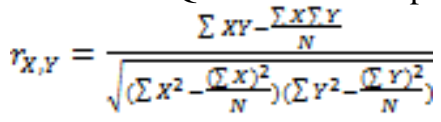

$\mathrm{X}$ means mean LQI, $\mathrm{Y}$ mean PRR value and $\mathrm{N}$ is the sample number. According to Eq. (11), $r_{X_{X} Y}=0.922$.

\section{Link Quality Estimation Model Based on imLQI}

According to the experiment results, we built a link quality estimation model by using different fitting models (Gaussian, Exponential, Smoothing, Spline, Power and Sum of Sine) to fit the relationship between mean LQI and PRR derived from imLQI. After comparison, the fitting goodness of Sum of Sine, which was 0.954, was better than that of other models, and the fitting result plots in Fig. 6. 


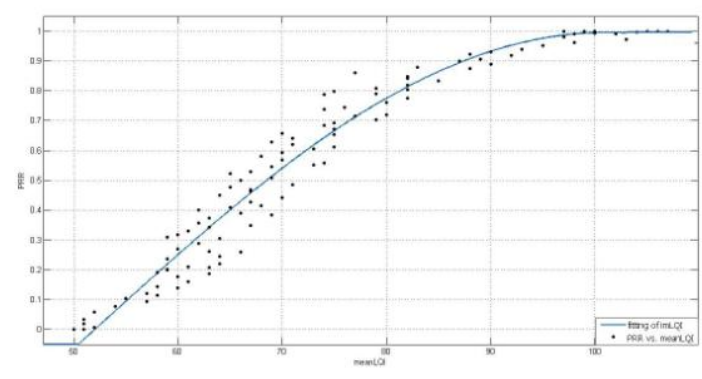

Figure 7. Revised curve fitting of mean LQI and PRR of imLQI

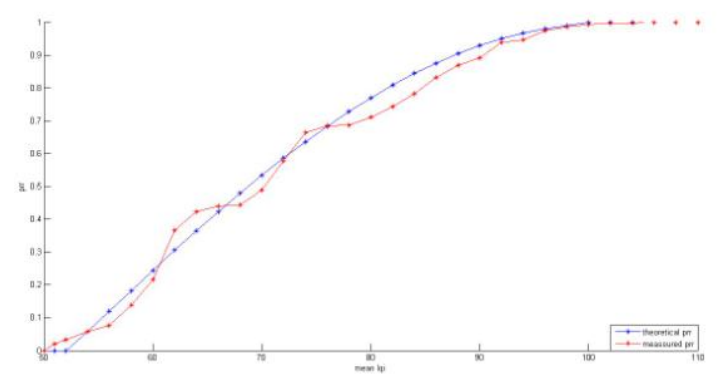

Figure 8. Comparison of calculated PRR from estimation model we built and measured PRR

The equation of Sum of Sine is

$$
\mathrm{Y}=\mathrm{a} 1{ }^{*} \sin (\mathrm{b} 1 * \mathrm{x}+\mathrm{c} 1)
$$

$X$ means mean LQI value, $Y$ means PRR value, and a1, b1, c1 are parameters. Therefore, Eq. (12) can be paraphrased to

$$
\mathrm{PRR}=\mathrm{a} 1 * \sin (\mathrm{b} 1 * \text { meanLQI+c } 1)
$$

In Fig.6, we notice that PRR drops when mean LQI is in $[100,110]$, and PRR is below 0 when mean LQI is in [50, 52]. In fact, PRR is usually above 0.95 when LQI is above 100 , and PRR is a value in $[0,1]$. The incorrectness in Fig 6 is brought by the characteristic of sine function. Hence, we revised the model to match reality:

$$
\left\{\begin{array}{lc}
P R R=1 & 100<L Q I \leq 110 \\
P R R=\left\{\frac{0.9958 * \sin (0.00118 * L Q I+4.26 \mathrm{a}}{0.95 * L Q I}\right\}, 52<L Q I \leq 100 & \\
P R R=0, & 50 \leq L Q I \leq 52
\end{array}\right.
$$

The fitting curve after revising is shown in Fig. 7.

By comparing the PRR values inferred from our estimation model with the measured values, we validated the correctness of our model. The comparison of calculated values and measured values plots in Fig. 8.

In Fig. 8, when PRR is in $[0.1,0.9]$, the discrepancy between the calculated value and measured value is respectively large, with the max discrepancy is $22 \%$ and the min discrepancy is $10 \%$. It is because links are unstable in the "transitional region", with large fluctuation.

According to statistics, the error rate distribution in our estimation model is shown in Fig. 9.

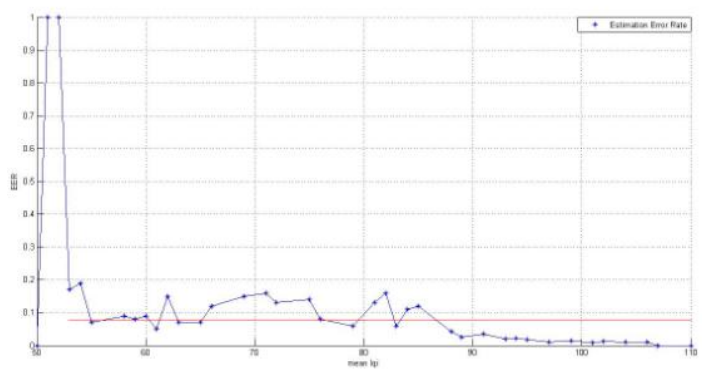

Figure 9. Error rate distribution

In our model, when mean LQI approaches to 50, the theoretical and measured PRR values are near 0 , so the error rate of our model in this region is 0 . When mean LQI is in [51, 52], we assume PRR is 0 , so the error rate is 1 . When mean LQI is in [53, 110], the mean estimation error rate is 0.0744, as the red line shown in Fig. 9, and the deviation is 0.0567. In general, the estimation we built on the basis of imLQI is with high correctness.

\section{CONCLUSION}

In Wireless Sensor Networks, an effective and accurate link quality estimation mechanism is important for the upper protocols and algorithms to achieve better performance. This article analyzes 3 different causes for packet loss by implementing measurements on testbed, and proposed an improved link quality estimation based on LQI called imLQI, which takes the LQI values of unsuccessfully received packets into the calculation of mean LQI. Meanwhile, we build a link quality estimation model on the basis of imLQI. Empirical results show that when using imLQI to compute mean LQI, PRR and mean LQI is more correlated compare with using traditional method in calculation. Besides, the link quality inferred from the estimation model we built is close to the measured results, which verified the correctness of our estimation model.

\section{REFERENCES}

[1] D. Couto, D. Aguayo, J. Bicket, and R. Morris. "A high throughput path metric for multihop wireless routing," In: Proceedings of the ACM MobiCom Conf., San Diego, CA, Sept. 2003.

[2] Gnawali O., Fonseca R., Jamieson K., Moss D., and Levis P."Collection Tree Protocol," In: Proceedings of the $7^{\text {th }}$ ACM Conference on Embedded Networked Sensor Systems (SenSys '09). ACM, pp: 90-100.2009.

[3] Zhao J. and Govindan R. "Understanding packet delivery performance in dense wireless sensor networks," In: Proceedings of the1st International Conference on Embedded Networked Sensor Systems (SenSys '03). ACM, pp: 1-13. 2003.

[4] Baccour N., et al. "Radio link quality estimation in wireless sensor networks: a survey," ACM Trans. Sen. Netw. Vol.8, No.4, Article 34. 2012.

[5] Senel M. ; Purdue Univ., Lafayette ; Chintalapudi, K. ; Lal, D. ; Keshavarzian, A. "A Kalman filter based link quality estimation scheme for wireless sensor networks," In: Proceedings of the IEEE Global Telecommunications Conference (GLOBECOM '07). IEEE, pp: 875-880. 2007 
[6] Kannan Srinivasan and Philip Levis. "RSSI is under appreciated," In: Proceedings of the 3rd Workshop on Embedded Networked Sensors (EmNets), 2006

[7] Jian, Z. and Z. Hai. "A link quality evaluation model in wireless sensor networks," In Proceedings of 3rd International Conference on Sensor Technologies and Applications (SENSORCOMM). pp: 1-5. 2009

[8] Jian, Z. and Z. Hai. "CCI-based evaluation model of wireless link," Journal of Northeastern University Natural Science, Vol. 29, pp: 1262-1265, 2008

[9] C. A. Boano, M. A. Zuniga, T. Voigt, A. Willig and K. Romer. "The triangle metric: fast link quality estimation for mobile wireless sensor networks," In: Proceedings of 19th International Conference on Computer Communications and Networks (ICCCN), pp: 1-7. 2010

[10] Yuan Zhengwu, Liang Junjun. "Cumulative link quality routing algorithm research on WSN. Computer Engineering and Applications," Vol. 47, No. 14. 2011

[11] J. Zhao and R. Govindan. "Understanding packet delivery performance in dense wireless sensor networks," In: Proceedings of the First ACM Conference on Embedded Network Sensor Systems, 2003

[12] Reijers, N., G. Halkes and K. Langendoen. "Link layer measurements in sensor networks," In: Proceedings of the $1^{\text {st }}$ IEEE International Conference on Mobile Adhoc and Sensor Systems, Oct, 2004, IEEE Computer Society Press. , 2004

[13] Shu Jian, Tao Juan, Liu Lin-lan, Chen Ye-bin, Zang Chao.. "CCIbased link quality estimation mechanism for wireless sensor networks under non-perceived packet loss," Journal of China University of Post and Telecommunications, Vol. 1, No.1, pp:1-10. 2013

[14] Kannan Srinivasan, Mayank Jain, Jung Il Choi, Tahir Azim, Edward S. Kim, Philip A. Levis, Bhaskar Krishnamachari. "The k factor: inferring protocol performance using inter-link reception correlation,' In: Proceedings of 16th Annual International Conference on Mobile Computing and Networking. pp: 317-328. 2010

[15] $\mathrm{CC} 1100$ Data Sheet http://focus.ti.com/docs/prod/folders/print/cc1100.html.

[16] Ashraf U, Abdellatif, Juanole G. "An interference and link quality aware routing metric for wireless mesh network,". IEEE $68^{\text {th }}$ Vehicular Technology Conference. IEEE Press. 2008

[17] Yuanqiang M.. "Improving wireless link delivery ratio classification with packet SNR," In: Proceedings of the International Conference on Electro Information Technology. IEEE, pp:6-12. 2005

[18] Sang L F, Arora A, Zhang H W. "On exploiting asymmetric wireless via one-way estimation,” In: Proceedings of MobiHoc. pp: 11-21. 2007

[19] Woo A. and Culler D.. "Evaluation of efficient link reliability estimators for low-power wireless networks," Tech. rep. UCB/CSD03-1270, EECS Department, University of California, Berkeley. 2003

[20] Kannan Srinivasan, Prabal Dutta, Arsalan Tavakoli and Philip Levis. "An empirical study of low power wireless," ACM Transactions on Sensor Networks (TOSN). Vol. 6, No. 16. 2010

[21] Zuniga M. and Krishnamachari B.. "An analysis of unreliability and asymmetry in low-power wireless links," ACM Trans. Sen. Netw. Vol. 3, No. 2, pp: 63-81. 2007 